Bull. Korean Math. Soc. 50 (2013), No. 4, pp. 1193-1200

http://dx.doi.org/10.4134/BKMS.2013.50.4.1193

\title{
INVARIANT RINGS AND REPRESENTATIONS OF SYMMETRIC GROUPS
}

\author{
Shotaro Kudo
}

\begin{abstract}
The center of the Lie group $S U(n)$ is isomorphic to $\mathbb{Z}_{n}$. If $d$ divides $n$, the quotient $S U(n) / \mathbb{Z}_{d}$ is also a Lie group. Such groups are locally isomorphic, and their Weyl groups $W\left(S U(n) / \mathbb{Z}_{d}\right)$ are the symmetric group $\Sigma_{n}$. However, the integral representations of the Weyl groups are not equivalent. Under the $\bmod p$ reductions, we consider the structure of invariant rings $H^{*}\left(B T^{n-1} ; \mathbb{F}_{p}\right)^{W}$ for $W=W\left(S U(n) / \mathbb{Z}_{d}\right)$. Particularly, we ask if each of them is a polynomial ring. Our results show some polynomial and non-polynomial cases.
\end{abstract}

Let $W$ be a finite group. For a modular representation

$$
\rho: W \longrightarrow G L\left(n ; \mathbb{F}_{p}\right),
$$

the group $\rho(W)$ acts on the polynomial algebra $S(V)=\mathbb{F}_{p}\left[t_{1}, \ldots, t_{n}\right]$. The set of invariants $S(V)^{\rho(W)}$ has a ring structure, and it is said to be the ring of invariants, [8] and [7]. In this paper, we discuss the invariant rings for various representations of the symmetric group $\Sigma_{n}$, along the line of work of [6].

The following is a topological aspect of our results, [8, Chapter 10]. Suppose $G$ is a compact connected Lie group. It is well-known that the cohomology of the classifying space $H^{*}(B G ; \mathbb{Q})$ is isomorphic to the ring of invariants $H^{*}\left(B T^{n} ; \mathbb{Q}\right)^{W(G)}$, which is a polynomial ring. We recall that $\mathbb{Q}$ can be replaced by a finite field $\mathbb{F}_{p}$ when the prime $p$ is large. Here we note $S(V) \cong H^{*}\left(B T^{n} ; \mathbb{F}_{p}\right)$.

We consider the integral representations of symmetric groups, which is the Weyl group of $S U(n)$. If $d$ divides $n$, the quotient $S U(n) / \mathbb{Z}_{d}$ is also a Lie group. The integral representations of $\Sigma_{n}$ induced by the actions of the Weyl groups of $S U(n) / \mathbb{Z}_{d}$ on maximal tori are $\mathbb{Z}$-inequivalent, [2]. In fact, the $\mathbb{Z}$-representation of $W\left(S U(n) / \mathbb{Z}_{d}\right)$ on $T^{n-1}$, up to $\mathbb{Z}$-equivalence, is given by $\phi_{d}^{-1} W(S U(n)) \phi_{d}$ for a non-singular matrix $\phi_{d}$. The representation of $\Sigma_{n}=W(S U(n))$ is generated

Received August 3, 2012; Revised October 5, 2012.

2010 Mathematics Subject Classification. Primary 55R35; Secondary 13A50, 55P60.

Key words and phrases. invariant theory, unstable algebra, pseudoreflection group, Lie group, $p$-compact group, classifying space. 
by the permutation matrices together with the following $(n-1) \times(n-1)$ matrix:

$$
\left(\begin{array}{cccc}
1 & & & -1 \\
& \ddots & & \vdots \\
& & 1 & \vdots \\
& & & -1
\end{array}\right) .
$$

In other words, each column vector is one of the set of the standard basis $\left\{\boldsymbol{e}_{1}, \boldsymbol{e}_{2}, \ldots, \boldsymbol{e}_{n-1}\right\}$ and the vector $\boldsymbol{b}={ }^{t}(-1,-1, \ldots,-1)$.

Let $W_{n, d}$ denote $\phi_{d}^{-1} W(S U(n)) \phi_{d}$. We use the same symbol for both integral and modular representations. If $p$ does not divide $d$, then $W_{n, d} \cong W(S U(n))$ at $p$. It is known that $H^{*}\left(B S U(n) ; \mathbb{F}_{p}\right)=H^{*}\left(B T^{n-1} ; \mathbb{F}_{p}\right)^{W_{n, 1}}$, and that $H^{*}\left(B T^{2} ; \mathbb{F}_{3}\right)^{W_{3,3}}$ is a polynomial ring which is not realizable, [5]. In the case of $n=4$ and $d=2$, the following result shows that the invariant ring $H^{*}\left(B T^{3} ; \mathbb{F}_{2}\right)^{W_{4,2}}$ is a polynomial ring which is realizable.

Theorem 1. Let $H^{*}\left(B T^{3} ; \mathbb{F}_{2}\right)=\mathbb{F}_{2}\left[t_{1}, t_{2}, t_{3}\right]$ with $\operatorname{deg}\left(t_{i}\right)=2$. The following hold:

(1) $H^{*}\left(B T^{3} ; \mathbb{F}_{2}\right)^{W_{4,2}}=\mathbb{F}_{2}\left[x_{2}, x_{4}, x_{6}\right]$, where $x_{2}=t_{3}, x_{4}=t_{1}^{2}+t_{2}^{2}+t_{1} t_{2}+$ $t_{1} t_{3}+t_{2} t_{3}$ and $x_{6}=t_{1} t_{2}\left(t_{1}+t_{2}+t_{3}\right)$.

(2) $H^{*}\left(B T^{3} ; \mathbb{F}_{2}\right)^{W_{4,2}} \cong H^{*}\left(B S U(3) \times B S^{1} ; \mathbb{F}_{2}\right)$.

We define the homomorphism $\rho_{d}: \Sigma_{4} \longrightarrow G L\left(3 ; \mathbb{F}_{2}\right)$ by $\rho_{d}(x)=\phi_{d}^{-1} x \phi_{d}$ for $x \in \Sigma_{4}$. Then $\operatorname{Im} \rho_{2}=W_{4,2}$ and $\operatorname{ker} \rho_{2} \cong \mathbb{Z}_{2}$. If $d=4$, on the other hand, then $\rho_{4}$ is a faithful representation. The structure of $H^{*}\left(B T^{4} ; \mathbb{F}_{2}\right)^{W_{4,4}}$ is as follows:

Theorem 2. For $n=d=4$, the following hold:

(1) $H^{*}\left(B T^{3} ; \mathbb{F}_{2}\right)^{W_{4,4}}=\mathbb{F}_{2}\left[x_{2}, x_{8}, x_{12}\right]$, where $x_{2}=t_{3}, x_{8}=t_{1}^{4}+t_{2}^{4}+t_{1}^{2} t_{2}^{2}+$ $t_{1}^{2} t_{3}^{2}+t_{2}^{2} t_{3}^{2}+t_{1}^{2} t_{2} t_{3}+t_{1} t_{2}^{2} t_{3}+t_{1} t_{2} t_{3}^{2}$ and $x_{12}=t_{1} t_{2}\left(t_{1}+t_{2}\right)\left(t_{1}+t_{3}\right)\left(t_{2}+t_{3}\right)\left(t_{1}+\right.$ $\left.t_{2}+t_{3}\right)$.

(2) $H^{*}\left(B T^{3} ; \mathbb{F}_{2}\right)^{W_{4,4}}$ is not realizable.

We note here that, in the case of $n=d$, the representation $W_{n, n}$ is equivalent to the dual representation $W(S U(n))^{*}$. It is known, [3], that for $p \geq 5$, the invariant ring $H^{*}\left(B T^{p-1} ; \mathbb{F}_{p}\right)^{W(S U(p))^{*}}$ is not polynomial. We will show some analogous results for $p=2,3$.

Theorem 3. The following hold:

(1) Let $n=6,8$. Then $H^{*}\left(B T^{n-1} ; \mathbb{F}_{2}\right)^{W_{n, n}}$ is not a polynomial ring.

(2) Let $n=6,9$. Then $H^{*}\left(B T^{n-1} ; \mathbb{F}_{3}\right)^{W_{n, n}}$ is not a polynomial ring.

We use a result of Dwyer-Wilkerson [3, Theorem 1.4]. Suppose that $V$ is a finite dimensional vector space over the field $\mathbb{F}_{p}$, and that $W$ is a subgroup of $\operatorname{Aut}(V)$. Let $U$ be a subset of $V$, and $W_{U}$ the subgroup of $W$ consisting of elements which fix $U$ pointwise. Then if $S(V)^{W^{*}}$ is a polynomial ring over $\mathbb{F}_{p}$, then $W_{U}$ must be a pseudoreflection group. 


\section{Invariant rings that are polynomial rings}

As sated in the introduction, the representation of $\Sigma_{n}=W(S U(n))$ is generated by the permutation matrices $\Sigma_{n-1}$ together with the following $(n-1) \times$ $(n-1)$ matrix:

$$
\left(\begin{array}{cccc}
1 & 0 & \cdots & -1 \\
0 & 1 & & -1 \\
\vdots & & \ddots & \vdots \\
0 & 0 & \cdots & -1
\end{array}\right)
$$

We write $W_{n, d}=\phi_{d}^{-1} W(S U(n)) \phi_{d}$ for the following matrix $\phi_{d}$ :

$$
\phi_{d}=\left(\begin{array}{cccc}
1 & & & 0 \\
& \ddots & & \vdots \\
& & 1 & 0 \\
d-1 & \cdots & d-1 & d
\end{array}\right) .
$$

We will prove Theorem 1 and Theorem 2 in this section. We consider the case of $n=4$. The representation $W(S U(4))$ is generated by 3 reflections.

$$
W(S U(4))=\Sigma_{4}=\left\langle\left(\begin{array}{lll}
0 & 1 & 0 \\
1 & 0 & 0 \\
0 & 0 & 1
\end{array}\right),\left(\begin{array}{lll}
1 & 0 & 0 \\
0 & 0 & 1 \\
0 & 1 & 0
\end{array}\right),\left(\begin{array}{lll}
1 & 0 & -1 \\
0 & 1 & -1 \\
0 & 0 & -1
\end{array}\right)\right\rangle .
$$

First take $d=2$. Notice that $\phi_{2}$ and $\phi_{2}^{-1}$ can be expressed as follows:

$$
\phi_{2}=\left(\begin{array}{lll}
1 & 0 & 0 \\
0 & 1 & 0 \\
1 & 1 & 2
\end{array}\right), \phi_{2}^{-1}=\left(\begin{array}{rrr}
1 & 0 & 0 \\
0 & 1 & 0 \\
-\frac{1}{2} & -\frac{1}{2} & \frac{1}{2}
\end{array}\right) .
$$

Consequently, the reflection group $W_{4,2}=\phi_{2}^{-1} W(S U(4)) \phi_{2}$ is generated by the following:

$$
W_{4,2}=\left\langle\left(\begin{array}{lll}
0 & 1 & 0 \\
1 & 0 & 0 \\
0 & 0 & 1
\end{array}\right),\left(\begin{array}{rrr}
1 & 0 & 0 \\
1 & 1 & 2 \\
-1 & 0 & -1
\end{array}\right),\left(\begin{array}{rrr}
0 & -1 & 2 \\
-1 & 0 & -2 \\
0 & 0 & 1
\end{array}\right)\right\rangle .
$$

Next consider the mod 2 reduction.

$$
W_{4,2}=\left\langle\left(\begin{array}{lll}
0 & 1 & 0 \\
1 & 0 & 0 \\
0 & 0 & 1
\end{array}\right),\left(\begin{array}{lll}
1 & 0 & 0 \\
1 & 1 & 0 \\
1 & 0 & 1
\end{array}\right)\right\rangle
$$

Hence, we see $W_{4,2} \cong \Sigma_{3}$ at $p=2$.

We recall how to see if a ring of invariants $H^{*}\left(B T^{n} ; \mathbb{F}_{p}\right)^{W}$ is polynomial, [4], [7] and [8]. A set of $n$ elements $x_{1}, x_{2}, \ldots, x_{n} \in H^{*}\left(B T^{n} ; \mathbb{F}_{p}\right)^{W}$ is said to be a 
system of parameters if the solution of the following system of equations

$$
\left\{\begin{array}{c}
x_{1}\left(t_{1}, t_{2}, \ldots, t_{n}\right)=0 \\
x_{2}\left(t_{1}, t_{2}, \ldots, t_{n}\right)=0 \\
\vdots \\
x_{n}\left(t_{1}, t_{2}, \ldots, t_{n}\right)=0
\end{array}\right.
$$

is trivial. Namely $t_{1}=t_{2}=\cdots=t_{n}=0$. As usual, we write $H^{*}\left(B T^{n} ; \mathbb{F}_{p}\right)=$ $\mathbb{F}_{p}\left[t_{1}, t_{2}, \ldots, t_{n}\right]$. Let $d(x)$ denote $\frac{1}{2} \operatorname{deg}(x)$ so that $d\left(t_{i}\right)=1$ for $1 \leq i \leq n$. According to [8, Proposition 5.5.5], for a finite group $W$, if we can find a system of parameters $\left\{x_{1}, x_{2}, \ldots x_{n}\right\}$ with $\prod_{i=1}^{n} d\left(x_{i}\right)=|W|$, then $H^{*}\left(B T^{n} ; \mathbb{F}_{p}\right)^{W}=$ $\mathbb{F}_{p}\left[x_{1}, x_{2}, \ldots x_{n}\right]$.

Proof of Theorem 1. (1) Suppose $x_{2}=t_{3}, x_{4}=t_{1}^{2}+t_{2}^{2}+t_{1} t_{2}+t_{1} t_{3}+t_{2} t_{3}$ and $x_{6}=t_{1} t_{2}\left(t_{1}+t_{2}+t_{3}\right)$. It is easy to check that the element $x_{2}, x_{4}, x_{6}$ are $W_{4,2}$-invariant, and that $\left\{x_{2}, x_{4}, x_{6}\right\}$ is a system of parameters. Consequently $H^{*}\left(B T^{3} \mathbb{F}_{2}\right)^{W_{4,2}}$ is the polynomial ring generated by $x_{2}, x_{4}$ and $x_{6}$, since $\left|W_{4,2}\right|=6=d\left(x_{2}\right) \cdot d\left(x_{4}\right) \cdot d\left(x_{6}\right)$.

(2) Recall that the mod 2 reduction of $W_{4,2}$ is generated by $A$ and $B$, where $A=\left(\begin{array}{lll}0 & 1 & 0 \\ 1 & 0 & 0 \\ 0 & 0 & 1\end{array}\right)$ and $B=\left(\begin{array}{lll}1 & 0 & 0 \\ 1 & 1 & 0 \\ 1 & 0 & 1\end{array}\right)$. Let $\psi=\left(\begin{array}{lll}1 & 1 & 0 \\ 1 & 0 & 0 \\ 0 & 1 & 1\end{array}\right)$. Then a calculation shows

$$
\bar{A}=\psi^{-1} A \psi=\left(\begin{array}{ccc}
1 & 1 & 0 \\
0 & 1 & 0 \\
0 & 0 & 1
\end{array}\right) \text { and } \bar{B}=\psi^{-1} B \psi=\left(\begin{array}{ccc}
0 & 1 & 0 \\
1 & 0 & 0 \\
0 & 0 & 1
\end{array}\right) \text {. }
$$

Hence, both matrices $\bar{A}$ and $\bar{B}$ are elements of $W(S U(3)) \times W\left(S^{1}\right)$. Notice that the mod 2 reduction of $W(S U(3))$ is generated by $\left(\begin{array}{ll}0 & 1 \\ 1 & 0\end{array}\right)$ and $\left(\begin{array}{ll}1 & 1 \\ 0 & 1\end{array}\right)$. This means that $W_{4,2}$ is equivalent to $W(S U(3)) \times W\left(S^{1}\right)$. Therefore, we see $H^{*}\left(B T^{3} ; \mathbb{F}_{2}\right)^{W_{4,2}} \cong H^{*}\left(B T^{3} ; \mathbb{F}_{2}\right)^{W(S U(3)) \times W\left(S^{1}\right)}=H^{*}\left(B S U(3) \times B S^{1} ; \mathbb{F}_{2}\right)$. This completes the proof.

Next consider the case of $d=4$. For $\phi_{4}=\left(\begin{array}{lll}1 & 0 & 0 \\ 0 & 1 & 0 \\ 3 & 3 & 4\end{array}\right)$, we see $W_{4,4}=$ $\phi_{4}^{-1} W(S U(4)) \phi_{4}$. The reflection group $W_{4,4}$ is generated by the matrices $\left(\begin{array}{lll}0 & 1 & 0 \\ 1 & 0 & 0 \\ 0 & 0 & 1\end{array}\right),\left(\begin{array}{ccc}1 & 0 & 0 \\ 3 & 3 & 4 \\ -3 & -2 & -3\end{array}\right)$ and $\left(\begin{array}{ccc}-2 & -3 & -4 \\ -3 & -2 & -4 \\ 3 & 3 & 5\end{array}\right)$. Hence its mod 2 reduction is as follows:

$$
W_{4,4}=\left\langle\left(\begin{array}{lll}
0 & 1 & 0 \\
1 & 0 & 0 \\
0 & 0 & 1
\end{array}\right),\left(\begin{array}{lll}
1 & 0 & 0 \\
1 & 1 & 0 \\
1 & 0 & 1
\end{array}\right),\left(\begin{array}{lll}
0 & 1 & 0 \\
1 & 0 & 0 \\
1 & 1 & 1
\end{array}\right)\right\rangle
$$

Proof of Theorem 2. (1) Notice that $\left\{x_{2}, x_{8}, x_{12}\right\}$ is a system of parameters. Furthermore $\left|W_{4,4}\right|=24=d\left(x_{2}\right) \cdot d\left(x_{8}\right) \cdot d\left(x_{12}\right)$. An argument similar to the one in Theorem 1 shows the desired result. 
(2) If the unstable algebra $H^{*}\left(B T^{3} ; \mathbb{F}_{2}\right)^{W_{4,4}}$ is realizable, there is a 2-compact group $X$ such that $H^{*}\left(B T^{3} ; \mathbb{F}_{2}\right)^{W_{4,4}} \cong H^{*}\left(B X ; \mathbb{F}_{2}\right)$. Since the polynomial algebra is generated by even-degree elements, the classifying space $B X$ is 2-torsion free. So the 2-adic cohomology is also a polynomial algebra generated by elements of the same degree. We can find, [1], a compact connected Lie group $G$ such that $H^{*}\left(B X ; \mathbb{Z}_{2}^{\wedge}\right) \cong H^{*}\left(B G ; \mathbb{Z}_{2}^{\wedge}\right)$. However, any Lie group $G$ does not satisfy the condition that $H^{*}\left(B G ; \mathbb{F}_{2}\right)=\mathbb{F}_{2}\left[x_{2}, x_{8}, x_{12}\right]$, since this cohomology does not contain a generator of degree 4 . Thus, $H^{*}\left(B T^{3} ; \mathbb{F}_{2}\right)^{W_{4,4}}$ is not realizable.

Remark 1.1. Recall that in the case of $n=d$, the representation $W_{n, n}$ is equivalent to the dual representation $W(S U(n))^{*}$. In fact, for the following $(n-1) \times(n-1)$ matrix,

$$
\phi=\left(\begin{array}{cccc}
2 & 1 & \cdots & 1 \\
1 & 2 & \cdots & 1 \\
\vdots & \vdots & \ddots & \vdots \\
1 & 1 & \cdots & 2
\end{array}\right)
$$

we see [5] that $\phi^{-1} \sigma \phi={ }^{t} \sigma$ for each of the generators $\sigma$ of the reflection group $\Sigma_{n-1}$. We claim that $\phi^{-1} \Sigma_{n-1} \phi=\Sigma_{n-1}^{*}$. Consequently, we see $\psi^{-1} W_{n, n} \psi=$ $W(S U(n))^{*}$ for $\psi=\phi_{n}^{-1} \phi$.

\section{Non-polynomial cases}

We will prove Theorem 3 in this section. To do so, we need a few basic results. As sated before, according to a result of Dwyer-Wilkerson, we will find a subset $U$ such that the subgroup $W_{U}$ is not generated by pseudoreflections. The representation $W(S U(n))$ as a subgroup of $G L\left(n-1, \mathbb{F}_{p}\right)$ is generated by the permutation representation of $\Sigma_{n-1}$ together with the following $(n-1) \times(n-1)$ matrix:

$$
\left(\begin{array}{cccc}
1 & & 0 & -1 \\
& \ddots & & \vdots \\
0 & & 1 & -1 \\
0 & \cdots & 0 & -1
\end{array}\right)
$$

In other words, if we let $\sum_{i=1}^{n} t_{i}=0$, then the representation of $W(S U(n))$ can be regarded as the permutation representation of $\Sigma_{n}$. For instance, when $n=4$, the transposition $(1,2)$ corresponds to the matrix $\left(\begin{array}{lll}0 & 1 & 0 \\ 1 & 0 & 0 \\ 0 & 0 & 1\end{array}\right),(2,3)$ to $\left(\begin{array}{lll}1 & 0 & 0 \\ 0 & 0 & 1 \\ 0 & 1 & 0\end{array}\right)$, and $(3,4)$ to $\left(\begin{array}{lll}1 & 0 & -1 \\ 0 & 1 & -1 \\ 0 & 0 & -1\end{array}\right)$, respectively. We will use this convention.

Proof of Theorem 3. (1) First we consider the case of $n=6$. Let $\boldsymbol{x}={ }^{t}(1,1,1$, $0,0)$ and $\boldsymbol{y}={ }^{t}(1,1,0,1,1)$, and let $U=\{\boldsymbol{x}, \boldsymbol{y}\}$. Recall that any element of $W(S U(6))$ is a $5 \times 5$ matrix such that each column is one of the set of the standard basis $\left\{\boldsymbol{e}_{1}, \boldsymbol{e}_{2}, \boldsymbol{e}_{3}, \boldsymbol{e}_{4}, \boldsymbol{e}_{5}\right\}$ and the vector $\boldsymbol{b}={ }^{t}(1,1,1,1,1)$, since $p=2$. 
Take a matrix $A=\left(\boldsymbol{a}_{1}, \boldsymbol{a}_{2}, \boldsymbol{a}_{3}, \boldsymbol{a}_{4}, \boldsymbol{a}_{5}\right) \in W(S U(6))$ such that $A \boldsymbol{x}=\boldsymbol{x}$. Notice that $A \boldsymbol{x}=\boldsymbol{a}_{1}+\boldsymbol{a}_{2}+\boldsymbol{a}_{3}$ and $\boldsymbol{x}=\boldsymbol{e}_{1}+\boldsymbol{e}_{2}+\boldsymbol{e}_{3}$. If the first column $\boldsymbol{a}_{1}$ is $\boldsymbol{e}_{1}$, then $\boldsymbol{b} \notin\left\{\boldsymbol{a}_{2}, \boldsymbol{a}_{3}\right\}$, and hence $\left\{\boldsymbol{a}_{2}, \boldsymbol{a}_{3}\right\}=\left\{\boldsymbol{e}_{2}, \boldsymbol{e}_{3}\right\}$. Similarly, if $\boldsymbol{a}_{1}=\boldsymbol{e}_{4}$, then one can show $\left\{\boldsymbol{a}_{2}, \boldsymbol{a}_{3}\right\}=\left\{\boldsymbol{e}_{5}, \boldsymbol{b}\right\}$. It turns out that all possible combinations are either

$$
\left\{\boldsymbol{a}_{1}, \boldsymbol{a}_{2}, \boldsymbol{a}_{3}\right\}=\left\{\boldsymbol{e}_{1}, \boldsymbol{e}_{2}, \boldsymbol{e}_{3}\right\} \text { and }\left\{\boldsymbol{a}_{4}, \boldsymbol{a}_{5}\right\} \subset\left\{\boldsymbol{e}_{4}, \boldsymbol{e}_{5}, \boldsymbol{b}\right\}
$$

or

$$
\left\{\boldsymbol{a}_{1}, \boldsymbol{a}_{2}, \boldsymbol{a}_{3}\right\}=\left\{\boldsymbol{e}_{4}, \boldsymbol{e}_{5}, \boldsymbol{b}\right\} \text { and }\left\{\boldsymbol{a}_{4}, \boldsymbol{a}_{5}\right\} \subset\left\{\boldsymbol{e}_{1}, \boldsymbol{e}_{2}, \boldsymbol{e}_{3}\right\} .
$$

Furthermore, we have $A \boldsymbol{y}=\boldsymbol{y}$ if $A \in W_{U}$. Again, notice that $A \boldsymbol{y}=\boldsymbol{a}_{1}+\boldsymbol{a}_{2}+$ $\boldsymbol{a}_{4}+\boldsymbol{a}_{5}$ and $\boldsymbol{y}=\boldsymbol{e}_{1}+\boldsymbol{e}_{2}+\boldsymbol{e}_{4}+\boldsymbol{e}_{5}$. One can show that $\boldsymbol{a}_{3}=\boldsymbol{e}_{3}$ or $\boldsymbol{b}$, and hence $W_{U} \cong D_{8}$ as follows:

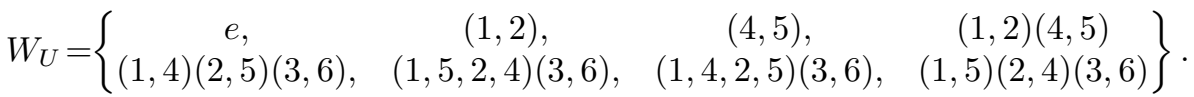

Here we regard, for example, as follows:

$$
(1,2)=\left(\boldsymbol{e}_{2}, \boldsymbol{e}_{1}, \boldsymbol{e}_{3}, \boldsymbol{e}_{4}, \boldsymbol{e}_{5}\right) \text { and }(1,5,2,4)(3,6)=\left(\boldsymbol{e}_{5}, \boldsymbol{e}_{4}, \boldsymbol{b}, \boldsymbol{e}_{1}, \boldsymbol{e}_{2}\right) .
$$

Since $W_{U}$ is not a pseudoreflection group, we see that $H^{*}\left(B T^{5} ; \mathbb{F}_{2}\right)^{W_{6,6}}$ is not a polynomial ring by [3].

Next consider the case of $n=8$. Let $U=\{\boldsymbol{x}, \boldsymbol{y}, \boldsymbol{z}\}$ for $\boldsymbol{x}={ }^{t}(1,1,1,1,0,0,0)$, $\boldsymbol{y}={ }^{t}(1,1,0,0,1,1,0)$ and $\boldsymbol{z}={ }^{t}(1,0,1,0,1,0,1)$. Any element of $W(S U(8))$ is a $7 \times 7$ matrix such that each column is one of the set of the standard basis $\left\{\boldsymbol{e}_{1}, \boldsymbol{e}_{2}, \ldots, \boldsymbol{e}_{7}\right\}$ and the vector $\boldsymbol{b}={ }^{t}(1,1,1,1,1,1,1)$. Take a matrix $A=\left(\boldsymbol{a}_{1}, \boldsymbol{a}_{2}, \ldots, \boldsymbol{a}_{7}\right) \in W(S U(8))$ such that $A \boldsymbol{x}=\boldsymbol{x}$. If the first column $\boldsymbol{a}_{1}$ is $\boldsymbol{e}_{1}$, then $\left\{\boldsymbol{a}_{2}, \boldsymbol{a}_{3}, \boldsymbol{a}_{4}\right\}=\left\{\boldsymbol{e}_{2}, \boldsymbol{e}_{3}, \boldsymbol{e}_{4}\right\}$. Similarly, if $\boldsymbol{a}_{1}=\boldsymbol{e}_{5}$, then $\left\{\boldsymbol{a}_{2}, \boldsymbol{a}_{3}, \boldsymbol{a}_{4}\right\}=$ $\left\{\boldsymbol{e}_{6}, \boldsymbol{e}_{7}, \boldsymbol{b}\right\}$. All possible combinations are either

$$
\left\{\boldsymbol{a}_{1}, \boldsymbol{a}_{2}, \boldsymbol{a}_{3}, \boldsymbol{a}_{4}\right\}=\left\{\boldsymbol{e}_{1}, \boldsymbol{e}_{2}, \boldsymbol{e}_{3}, \boldsymbol{e}_{4}\right\} \text { and }\left\{\boldsymbol{a}_{5}, \boldsymbol{a}_{6}, \boldsymbol{a}_{7}\right\} \subset\left\{\boldsymbol{e}_{5}, \boldsymbol{e}_{6}, \boldsymbol{e}_{7}, \boldsymbol{b}\right\}
$$

or

$$
\left\{\boldsymbol{a}_{1}, \boldsymbol{a}_{2}, \boldsymbol{a}_{3}, \boldsymbol{a}_{4}\right\}=\left\{\boldsymbol{e}_{5}, \boldsymbol{e}_{6}, \boldsymbol{e}_{7}, \boldsymbol{b}\right\} \text { and }\left\{\boldsymbol{a}_{5}, \boldsymbol{a}_{6}, \boldsymbol{a}_{7}\right\} \subset\left\{\boldsymbol{e}_{1}, \boldsymbol{e}_{2}, \boldsymbol{e}_{3}, \boldsymbol{e}_{4}\right\} .
$$

Furthermore, we have $A \boldsymbol{y}=\boldsymbol{y}$ and $A \boldsymbol{z}=\boldsymbol{z}$ if $A \in W_{U}$. One can show that $W_{U}$ is expressed as follows:

$$
W_{U}=\mathbb{Z} / 2\langle\alpha\rangle \times \mathbb{Z} / 2\langle\beta\rangle \times \mathbb{Z} / 2\langle\gamma\rangle,
$$

where $\alpha=(1,2)(3,4)(5,6)(7,8), \beta=(1,3)(2,4)(5,7)(6,8)$ and $\gamma=(1,5)(2,6)$ $(3,7)(4,8)$. This group is not a pseudoreflection group, hence $H^{*}\left(B T^{7} ; \mathbb{F}_{2}\right)^{W_{8,8}}$ is not a polynomial ring.

(2) We consider the case of $n=6$ at $p=3$. Let $U=\{\boldsymbol{x}, \boldsymbol{y}\}$ for $\boldsymbol{x}=$ ${ }^{t}(1,1,-1,-1,0)$ and $\boldsymbol{y}=(1,-1,0,1,-1)$. Take a matrix $A=\left(\boldsymbol{a}_{1}, \boldsymbol{a}_{2}, \boldsymbol{a}_{3}, \boldsymbol{a}_{4}, \boldsymbol{a}_{5}\right)$ $\in W(S U(6))$ such that $A \boldsymbol{x}=\boldsymbol{x}$. It the first column $\boldsymbol{a}_{1}$ is $\boldsymbol{e}_{1}$, then $\boldsymbol{a}_{2}=\boldsymbol{e}_{2}$. Similarly, if $\boldsymbol{a}_{1}=\boldsymbol{e}_{3}$, then $\boldsymbol{a}_{2}=\boldsymbol{e}_{4}$, and if $\boldsymbol{a}_{1}=\boldsymbol{e}_{5}$, then $\boldsymbol{a}_{2}=\boldsymbol{b}$. Here $\boldsymbol{b}={ }^{t}(-1,-1,-1,-1,-1)$. One of the following holds:

$$
\left\{\boldsymbol{a}_{1}, \boldsymbol{a}_{2}\right\}=\left\{\boldsymbol{e}_{1}, \boldsymbol{e}_{2}\right\},\left\{\boldsymbol{a}_{3}, \boldsymbol{a}_{4}\right\}=\left\{\boldsymbol{e}_{3}, \boldsymbol{e}_{4}\right\} \text { and } \boldsymbol{a}_{5} \in\left\{\boldsymbol{e}_{5}, \boldsymbol{b}\right\}
$$


or

$$
\left\{\boldsymbol{a}_{1}, \boldsymbol{a}_{2}\right\}=\left\{\boldsymbol{e}_{3}, \boldsymbol{e}_{4}\right\},\left\{\boldsymbol{a}_{3}, \boldsymbol{a}_{4}\right\}=\left\{\boldsymbol{e}_{5}, \boldsymbol{b}\right\} \text { and } \boldsymbol{a}_{5} \in\left\{\boldsymbol{e}_{1}, \boldsymbol{e}_{2}\right\}
$$

or

$$
\left\{\boldsymbol{a}_{1}, \boldsymbol{a}_{2}\right\}=\left\{\boldsymbol{e}_{5}, \boldsymbol{b}\right\},\left\{\boldsymbol{a}_{3}, \boldsymbol{a}_{4}\right\}=\left\{\boldsymbol{e}_{1}, \boldsymbol{e}_{2}\right\} \text { and } \boldsymbol{a}_{5} \in\left\{\boldsymbol{e}_{3}, \boldsymbol{e}_{4}\right\} .
$$

Furthermore, we have $A \boldsymbol{y}=\boldsymbol{y}$ if $A \in W_{U}$. It follows that

$$
W_{U}=\mathbb{Z} / 3\langle\alpha\rangle,
$$

where $\alpha=(1,3,5)(2,4,6)$. Therefore, this group is not a pseudoreflection group, and hence $H^{*}\left(B T^{5} ; \mathbb{F}_{3}\right)^{W_{6,6}}$ is not a polynomial ring.

Next consider the case of $n=9$. Let $U=\{\boldsymbol{x}, \boldsymbol{y}\}$ for $\boldsymbol{x}={ }^{t}(1,1,1,-1,-1,-1$, $0,0)$ and $\boldsymbol{y}={ }^{t}(1,-1,0,1,-1,0,1,-1)$. Take a matrix $A=\left(\boldsymbol{a}_{1}, \boldsymbol{a}_{2}, \ldots, \boldsymbol{a}_{8}\right) \in$ $W(S U(9))$ such that $A \boldsymbol{x}=\boldsymbol{x}$. It the first column $\boldsymbol{a}_{1}$ is $\boldsymbol{e}_{1}$, then we obtain $\left\{\boldsymbol{a}_{2}, \boldsymbol{a}_{3}\right\}=\left\{\boldsymbol{e}_{2}, \boldsymbol{e}_{3}\right\}$. Similarly, if $\boldsymbol{a}_{1}=\boldsymbol{e}_{4}$, then $\left\{\boldsymbol{a}_{2}, \boldsymbol{a}_{3}\right\}=\left\{\boldsymbol{e}_{5}, \boldsymbol{e}_{6}\right\}$, and if $\boldsymbol{a}_{1}=\boldsymbol{e}_{7}$, then $\left\{\boldsymbol{a}_{2}, \boldsymbol{a}_{3}\right\}=\left\{\boldsymbol{e}_{8}, \boldsymbol{b}\right\}$. Here $\boldsymbol{b}={ }^{t}(-1,-1,-1,-1,-1,-1,-1,-1)$. We see that one of the following holds:

$\left\{\boldsymbol{a}_{1}, \boldsymbol{a}_{2}, \boldsymbol{a}_{3}\right\}=\left\{\boldsymbol{e}_{1}, \boldsymbol{e}_{2}, \boldsymbol{e}_{3}\right\},\left\{\boldsymbol{a}_{4}, \boldsymbol{a}_{5}, \boldsymbol{a}_{6}\right\}=\left\{\boldsymbol{e}_{4}, \boldsymbol{e}_{5}, \boldsymbol{e}_{6}\right\}$ and $\left\{\boldsymbol{a}_{7}, \boldsymbol{a}_{8}\right\} \subset\left\{\boldsymbol{e}_{7}, \boldsymbol{e}_{8}, \boldsymbol{b}\right\}$ or

$\left\{\boldsymbol{a}_{1}, \boldsymbol{a}_{2}, \boldsymbol{a}_{3}\right\}=\left\{\boldsymbol{e}_{4}, \boldsymbol{e}_{5}, \boldsymbol{e}_{6}\right\},\left\{\boldsymbol{a}_{4}, \boldsymbol{a}_{5}, \boldsymbol{a}_{6}\right\}=\left\{\boldsymbol{e}_{7}, \boldsymbol{e}_{8}, \boldsymbol{b}\right\}$ and $\left\{\boldsymbol{a}_{7}, \boldsymbol{a}_{8}\right\} \subset\left\{\boldsymbol{e}_{1}, \boldsymbol{e}_{2}, \boldsymbol{e}_{3}\right\}$ or

$\left\{\boldsymbol{a}_{1}, \boldsymbol{a}_{2}, \boldsymbol{a}_{3}\right\}=\left\{\boldsymbol{e}_{7}, \boldsymbol{e}_{8}, \boldsymbol{b}\right\},\left\{\boldsymbol{a}_{4}, \boldsymbol{a}_{5}, \boldsymbol{a}_{6}\right\}=\left\{\boldsymbol{e}_{1}, \boldsymbol{e}_{2}, \boldsymbol{e}_{3}\right\}$ and $\left\{\boldsymbol{a}_{7}, \boldsymbol{a}_{8}\right\} \subset\left\{\boldsymbol{e}_{4}, \boldsymbol{e}_{5}, \boldsymbol{e}_{6}\right\}$.

Furthermore, we have $A \boldsymbol{y}=\boldsymbol{y}$ if $A \in W_{U}$. Consequently, we see that

$$
W_{U}=\mathbb{Z} / 3\langle\alpha\rangle \times \mathbb{Z} / 3\langle\beta\rangle,
$$

where $\alpha=(1,2,3)(4,5,6)(7,8,9)$ and $\beta=(1,4,7)(2,5,8)(3,6,9)$. Once again $H^{*}\left(B T^{8} ; \mathbb{F}_{3}\right)^{W_{9,9}}$ is not a polynomial ring.

Remark 2.1. In the proof of Theorem 3, we have found subgroups $W_{U}$ which are not pseudoreflection groups. In the case of $n=6$ and $p=2$, the group $W_{U}$ can be made smaller. Namely, if $\boldsymbol{z}={ }^{t}(1,0,1,0,1)$ and $U=\{\boldsymbol{x}, \boldsymbol{y}, \boldsymbol{z}\}$, then $W_{U}$ can be expressed as follows:

$$
W_{U}=\mathbb{Z} / 2\langle\alpha\rangle
$$

where $\alpha=(1,4)(2,5)(3,6)$. This is not a pseudoreflection group.

\section{References}

[1] K. K. S. Andersen and J. Grodal, The classification of 2-compact groups, J. Amer. Math. Soc. 22 (2009), no. 2, 387-436

[2] M. Craig, A characterization of certain extreme forms, Illinois J. Math. 20 (1976), no. 4, 706-717.

[3] W. G. Dwyer and C. W. Wilkerson, Kähler differentials, the T-functor, and a theorem of Steinberg, Trans. Amer. Math. Soc. 350 (1998), no. 12, 4919-4930.

[4] - Poincaré duality and Steinberg's theorem on rings of coinvariants, Proc. Amer. Math. Soc. 138 (2010), no. 10, 3769-3775. 
[5] K. Ishiguro, Projective unitary groups and K-theory of classifying spaces, Fukuoka Univ. Sci. Rep. 28 (1998), no. 1, 1-6.

[6] _ Invariant rings and dual representations of dihedral groups, J. Korean Math. Soc. 47 (2010), no. 2, 299-309.

[7] R. M. Kane, Reflection Groups and Invariant Theory, CMS Books in Mathematics/Ouvrages de Mathématiques de la SMC, 5. Springer-Verlag, 2001.

[8] L. Smith, Polynomial Invariants of Finite Groups, A. K. Peters, Ltd., Wellesley, MA, 1995.

FUKUOKA UNIVERSITY

FUKUOKA 814-0180, JAPAN

E-mail address: sd100501@cis.fukuoka-u.ac.jp 